\title{
EDITORIAL
}

\section{Retiring the Learning Curve}

\author{
Henry Buchwald • Nicola Scopinaro
}

Published online: 1 April 2009

(C) Springer Science + Business Media, LLC 2009

Tom Chalmers was a great American advocate for the power of metaanalyses and the adjudication of doubt through randomized controlled trials. He was famous for his admonition to randomize with the first patient in a trial and for his abhorrence of the learning curve in clinical therapeutics. A scientific icon, Dr. Chalmers was an internist, a gastroenterologist, a NIH researcher, a department head, a medical school dean, and a hospital president. His distaste for the learning curve principle was universal and not limited to surgery. He often stated that if a physician or surgeon believed that his first cohort of patients undergoing a new procedure would have a higher mortality and morbidity than his/her subsequent patients, the doctor was obligated to share that perception with those first patients and to tell them that his care of them will be more filled with hazard than he hopes his subsequent efforts will be.

In our current era of disclosure, should not this transparency of information be expected from all physicians and surgeons? The only reasonable alternative to such full disclosure in the practice of bariatric surgery is to retire in principle and, more importantly, in reality, the "learning curve" concept.

The paradox of our field is this: On the one hand, bariatric surgery requires rigorous, specialized training and the repetitive practice that begets mastery. On the other

H. Buchwald ( $\square)$

University of Minnesota,

Minneapolis, MN, USA

e-mail: buchw001@umn.edu

N. Scopinaro

University of Genoa,

Genoa, Italy hand, there are no techniques to learn that are, in and of themselves, novel.

The cutting, dissecting, resecting, sewing, and stapling, as well as the clamping, ligating, and cauterizing or sealing of vessels, lysis of adhesions, and even the insertion of foreign bodies, require the same skills as do other aspects of abdominal surgery. Gaining access to the abdominal cavity laparoscopically or by open surgery is also the same, except that the abdominal wall is thicker in the obese patient. Bariatric operations are, in fact, only the combining of these basic surgical acts to perform a particular procedure. Further, all these basic techniques should be learned and mastered during a 5-year residency. We grant that gaining exposure for bariatric surgery is more difficult, especially in the attic of the abdomen. Obtaining adequate exposure in the obese patient needs to be observed and practiced. There are training programs today in which bariatric surgery is actually used to teach general abdominal surgery and not vice versa.

Entering any specialty discipline requires proceeding with caution. After bariatric training, the novice bariatric surgeon should start with patients who are neither super obese nor have extraordinary comorbidities (e.g., patients on a kidney, liver, or heart transplant list), as well as abstaining from revisional surgery until primary bariatric surgery has been mastered.

The major complications of bariatric surgery for which the concept of the learning curve has been used as an excuse for suboptimal results include death, leaks, hemorrhage, and postoperative misadventures. Let us consider them in reverse order.

Postoperative misadventures, e.g., myocardial infarctions, deep venous thrombosis and pulmonary emboli, out-of-control blood sugar levels, wound infections, pulmonary complications, urinary tract problems, and pro- 
longed ileus, are often associated with inadequate preoperative cardiovascular, blood pressure, respiratory, and diabetes assessment and management; lack of or injudicious use of intraoperative antibiotics, anticoagulants, and beta blockers; and postoperative neglect of adequate pulmonary toilet, ambulation, and routine fluid, electrolyte, blood insulin, and pain management. These aspects of patient management are elementary and, in essence, have nothing to do with performing bariatric surgery.

As a rule, the majority of postoperative misadventures are failures of intraoperative technique, such as rough tissue handling, causing excessive bleeding and other surgical errors. A smooth postoperative course is dictated by events during, not after, what takes place in the operating room.

Intraoperative blood loss increases the incidence of postoperative complications and is correlated with postoperative mortality. Blood loss, especially during a primary bariatric procedure, should be absolutely minimal. Though a massive intraoperative bleed will occur in the experience of the best surgeon, such bleeds should be an anomaly, and the use of blood replacement in a primary bariatric procedure should be most rare. The need to return to the operating room postoperatively for bleeding is usually determined intraoperatively.

Surgeons often cite a reduction in their leak rate as the primary benefit of their steep learning curve for bariatric surgery. Postoperative leaks, however, are created in the operating room and are not due to performing bariatric surgery in and of itself. Leaks represent poor anastomotic technique per se and are caused by a failure to oversew staple lines, using coarser than what is appropriate or necessary suture material, sloppy placement of sutures, and, all too often, haste. Leaks, like blood loss, should be rare and should be rare from the outset of a career in bariatric surgery. To perform a leak-free anastomosis, open or laparoscopically, is elementary general surgery, not an acquired skill of bariatric surgery.

Finally, postoperative deaths do not usually occur due to chance. They are the cumulative result of leaks, hemorrhage, and postoperative misadventures, and other, usually avoidable, complications. Since the timing for bariatric surgery is always elective, there is no excuse for inadequate patient preparation. Bariatric patients have many chronic comorbidities, but they are not debilitated, usually not protein- or vitamin-depleted, and not burdened by cancer. A bariatric patient should not die as a direct result of the surgery. Though there will be deaths in bariatric surgery, their incidence rate should be well under $0.5 \%$.

Over time and with experience, the maturing bariatric surgeon will perform a given operation in less time. We see increased proficiency, not surgical mishaps, as the only legitimate designation for a learning curve. Speed, however, should never be the yardstick for measuring competence. Senior surgeons speak of their time skin-to-skin. It would be well instead to focus on and to teach gentleness and precision in handling tissues, successful outcomes, and low mortality and morbidity rates. Such accomplishments are the true standards for a role model. Such accomplishments come with being a good surgeon and do not require a learning curve.

We would recommend that a surgeon interested in a career in bariatrics obtain the appropriate training and mentoring. In the 1950s and 1960s, few surgical trainees would escape learning the basics of peptic ulcer surgery. The same situation with regard to learning the basics of bariatric surgery exists today in many general surgery residency training programs. Five years of surgical residency provide a young surgeon with the fundamentals of our craft, and bariatric surgery fellowships provide valuable opportunities for postgraduate specialty training.

Additionally, for the practicing general surgeon wishing to enter this field, a variety of mentoring programs are available. With the evolution from bariatric to bariatric/ metabolic surgery, there may even be a specialty board examination or certification requirement for this discipline. At present, qualifying standards for centers of excellence, with surgeons of excellence, have been set by the Surgical Review Corporation, its American College of Surgeons equivalent, and a fledgling European consortium.

The rigorous training of general surgeons and, subsequently, of bariatric/metabolic surgeons and our determination to maintain high standards for our discipline will retire the idea that there must be a learning curve and ensure that no patient will be offered less than optimal results. 